\title{
Monte Carlo simulation of particle-induced bit upsets
}

\author{
Frédéric Wrobel $^{1,{ }^{*}}$, Antoine Touboul ${ }^{1}$, Jean-Roch Vaillé ${ }^{1,2}$, Jérôme Boch ${ }^{1}$, and Frédéric Saigné ${ }^{1}$ \\ ${ }^{1}$ Université de Montpellier, Institut d'Electronique et des Systèmes, UMR-CNRS 5214, France \\ ${ }^{2}$ Université de Nîmes, France
}

\begin{abstract}
We investigate the issue of radiation-induced failures in electronic devices by developing a Monte Carlo tool called MC-Oracle. It is able to transport the particles in device, to calculate the energy deposited in the sensitive region of the device and to calculate the transient current induced by the primary particle and the secondary particles produced during nuclear reactions. We compare our simulation results with SRAM experiments irradiated with neutrons, protons and ions. The agreement is very good and shows that it is possible to predict the soft error rate (SER) for a given device in a given environment.
\end{abstract}

\section{Introduction}

Radiation-induced failures in electronic devices represent a major concern for microelectronic reliability, especially for aerospace applications [1-3]. In satellites, devices are exposed to protons, electrons and heavy ions leading to malfunctions. In the following, we will consider a specific kind of malfunction that is called single event effect (SEE), meaning that a unique particle is able to trigger a failure. The cosmic particles that are at play are protons and heavy ions. Moreover, cosmic rays are able to interact with molecule nucleus of the atmosphere and produce secondary particles whose flux depends on the altitude. Fig. 1 gives the typical particle flux calculated in the atmosphere as a function of altitude with QARM [4]. Even if different kinds of particles exist, the main contribution to the SEE rate in atmosphere is actually attributed to neutrons.

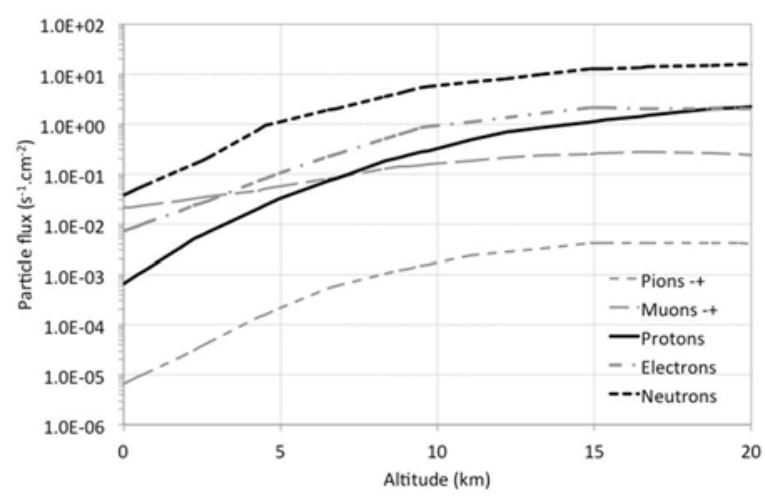

Figure 1. Particles fluxes in the atmosphere as a function of altitude (simulated with QARM).

\footnotetext{
` Corresponding author: frederic.wrobel@umontpellier.fr
}

One major issue for memory devices is when a unique particle is able to upset a bit from its value to its opposite. These Single Event Upsets (SEU) can occur in space and also in atmosphere and it is useful to be able to predict the number of failures induced in a given memory at a given location (i.e. as a function of altitude and latitude). To do so, we developed the Monte Carlo code MCORACLE. This code simulates the passage of particles in a complex structure composed of multiple volumes with various materials (silicon, silicon dioxide, metals...). Secondary ions produced during nuclear reactions are treated in the energy range of $1 \mathrm{MeV}-200 \mathrm{MeV}$, which is representative of the atmospheric spectrum. The ionizing particles are tracked in the structure and the released energy is determined in the region of the device that is known to be sensitive. Using an SEU criterion it is then possible to determine whether an error occurs or not. Bit flip cross sections are determined as a function of incident energy or as a function of ion stopping power. The Soft Error Rate can also be calculated for a specific location, which is defined by the altitude and the latitude in atmosphere or for a given mission in Space.

\section{The Monte Carlo approach}

We developed the MC-Oracle code, for which a first simplified version has been presented in [5]. MC-Oracle is a Monte Carlo tool that allows investigating Single Event Upsets (SEU), Multiple Cell Upsets (MCU), and Single Event Transients (SET). The bit flip cross sections as well as the soft error rate (SER) are the main quantities that are provided by the code.

Fig. 2 represents the flowchart of the code, which 
shows three main parts: the structure definition, the particle-structure interaction and the effect on the electronic device.

First, we need to define the geometry and the materials that compose the device. We also need to give the location of the electrodes transistors, as they will be able to collect the charge deposited in the structure by the ionizing particles. Moreover, it is necessary to define the kind of particles that interact with the device (neutrons from atmosphere, or protons or ions from space).

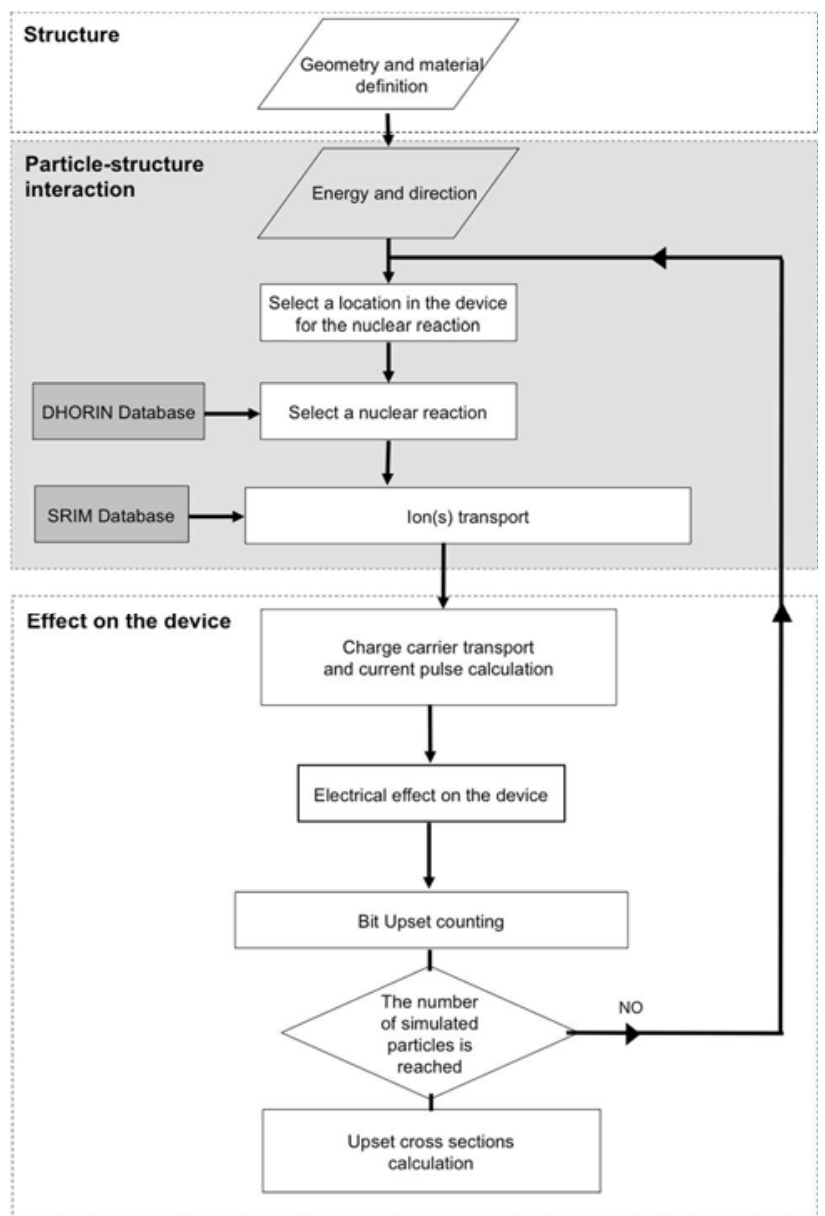

Figure 2. Flowchart of the Monte Carlo tool (MC-Oracle)

Once the definition of the simulation is done, MCOracle starts the simulation with a random choice of an incident particle, its position, energy and direction. Obviously, this is done with respect to the distributions of energy and direction. The initial position is selected outside the device. For incident ions only ionization is accounted for as it has already been established that nuclear reactions induced by ions have a very low contribution to upset bit rate.

For incident neutron and proton, nuclear reactions have been pre-calculated with the DHORIN code [6]. This latest uses the model of $2 \mathrm{D}$-exciton model for preequilibrium step, the formulation of Hauser-Feshbach and the generalized evaporation model. Elastic reactions are treated with the ECIS code that uses spherical optical models from the RIPL2 database. The DHORIN database is composed of several monoenergetic simulation files that contain millions of nuclear reactions details about the secondary ions (nature, energy and direction). These ionizing particles are transported within the device and the deposition of energy is calculated in the sensitive regions using the SRIM database made of the stopping power of various ions as a function of energy [7].

Next, the effect of the energy deposition in the device is treated. To do so, the tool divides each ion track into small fragments that spherically diffuse their charge carriers and can be collected by the drain electrode that is itself divided into elemental sections. Then, integrating the charge diffusion along the whole track and over the whole drain surface gives the current pulse that is generated by the nuclear reaction at the electrode of each transistor. A basic electrical model of the memory cell is finally used to state whether the calculated transient current is able to trigger a cell upset or not.

In our simple study, the 3D-simulated device structure is a bulk made of a silicon layer with a surface of $30 \mu \mathrm{m}$ x $30 \mu \mathrm{m}$ and a thickness of $20 \mu \mathrm{m}$. Above this bulk, we added a layer of $10 \mu \mathrm{m}$ of silicon dioxide to account for the Back End Of Line (BEOL). At the $\mathrm{Si} / \mathrm{SiO}_{2}$ interface, we simulate $20 \times 20$ SRAM cells, each bit being modeled by 4 transistors (we did not account for the pass gate transistors that are less sensitive). For each cell we have two OFF transistors (one NMOS and one PMOS), which are likely to collect the charge.

The SEU cross section is finally evaluated by calculating the ratio of the number of bit flips and the simulated particle fluence. In the case of nonmonoenergetic incident particles, the spectrum can be provided to the code. In that case, it is more convenient to calculate the soft error rate (SER), which directly account for the real environment.

\section{Results}

In this section we present some results obtained with MC-ORACLE for devices irradiated with heavy ion, proton and neutron. Figure 3 shows the comparison between the Monte Carlo approach and the experimental results for a $65 \mathrm{~nm}$ SRAM irradiated with heavy ions [8]. The calculated Single Event Bit upset cross section is in good agreement with experimental results above 0.5 $\mathrm{MeV} . \mathrm{cm}^{2} / \mathrm{mg}$ which shows that the modeling is efficient. At lower LET a singular peak is observed and is predicted by the Monte Carlo tool, though the order of magnitude is not correct. This peak corresponds to irradiation with protons for which the ionization is efficient to trigger SEU very near the Bragg's peak. 


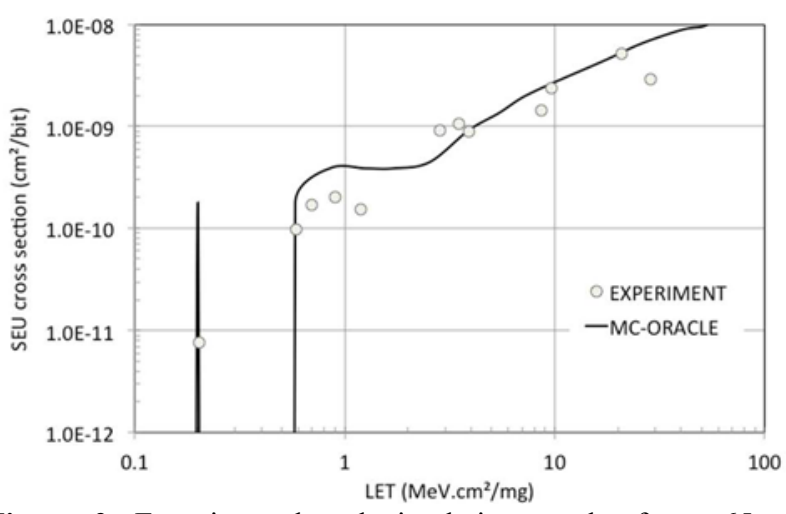

Figure 3. Experimental and simulation results for a $65 \mathrm{~nm}$ SRAM irradiated with ions.

Figure 4 shows the comparison between the Monte Carlo approach and the experimental results for a $90 \mathrm{~nm}$ SRAM irradiated with protons [9] and as a function of proton energy. The calculated cross section is in good agreement with the experimental data. In figure 5, we plotted the results obtained with the $90 \mathrm{~nm}$ SRAM irradiated with neutrons. Neutrons are here not monoenergetic and the energy distribution has actually the same shape than the atmospheric spectrum. The results are then given in FIT/Mbit, one Failure In Time being the number of failures during one billion of hours. The soft error rate is calculated for different supply voltage $V_{D D}$ that are characteristic of the normal functioning of the device. Here again the simulation results are in good agreement with experimental data.

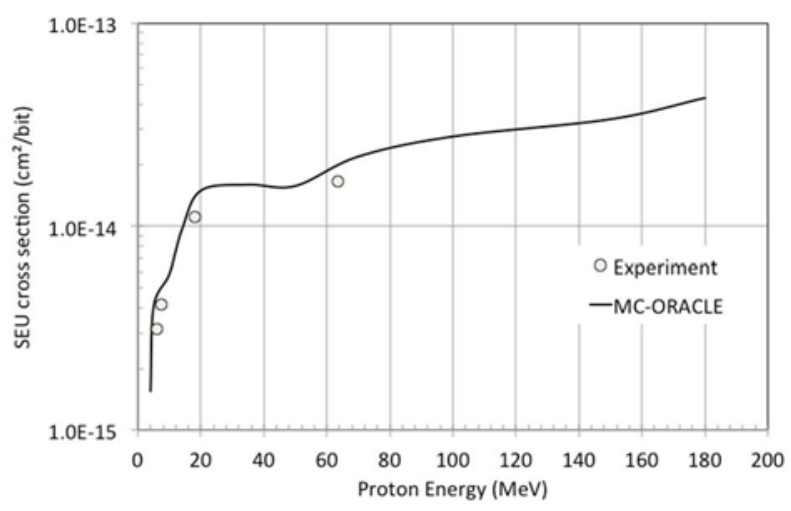

Figure 4. Experimental and simulation results for a $90 \mathrm{~nm}$ SRAM irradiated with protons.

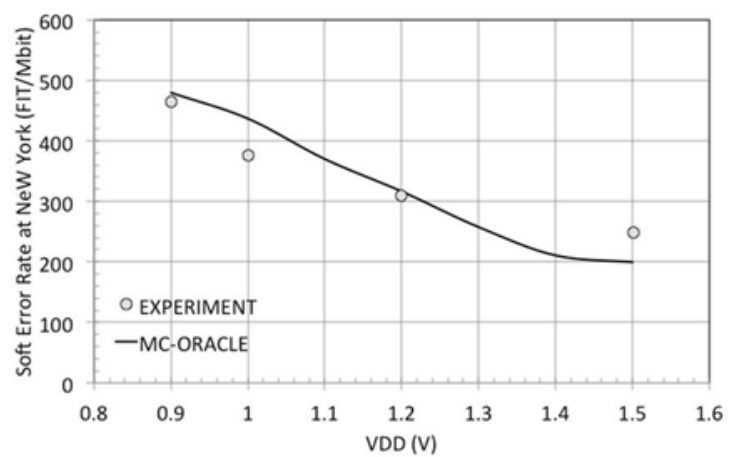

Figure 5. Experimental and simulation results for a $90 \mathrm{~nm}$ SRAM irradiated with neutrons for various voltages.

\section{Summary and conclusions}

In order to address the issue of radiation-induced failures in electronic devices we developed the MCOracle tool. This code is able to transport the particles within the device and, based on the energy deposition, is able to calculate the transient current induced by the primary particle and the secondary particles produced during nuclear reactions.

We show that our approach is consistent by comparing SRAM experiments under neutron, proton and ion with our simulations results. The agreement is very good and shows that it is possible to predict the soft error rate (SER) for a given device in a given environment.

\section{References}

1. R. Baumann, "Soft errors in advanced computer systems," IEEE Trans. Device Mater. Rel., vol. 22, no. 3, pp. 258-266, May-Jun. 2005.

2. T. Karnik and P. Hazucha, "Characterization of soft errors caused by single event upsets in CMOS processes," IEEE Trans. Depend. Secure Comput., vol. 1, no. 2, pp. 128-143, Apr.-Jun. 2004

3. R. D. Schrimpf, K. M. Warren, R. A. Weller, R. A. Reed, L. W. Massengill, M. L. Alles, D. M. Fleetwood, X. J. Zhou, L. Tsetseris, and S.T. Pantelides, "Reliability and radiation effects in IC technologies," in Proc. IEEE Int. Reliability Physics Symp., Apr. 27-May 1 2008, pp. 97-106.

4. F.Lei,A.Hands,S.Clucas,C.Dyer,andP.Truscott,"Improvemen tsto and validations of the QinetiQ atmospheric radiation model (QARM)," in Proc. 8th Eur. Conf. Radiation and Its Effects on Components and Systems, 2005, pp. D3-1-D3-8.

5. F. Wrobel, F. Saigné, « MC-ORACLE: A tool for predicting Soft Error Rate ", Computer Physics Communications 182 (2011), 317-321

6. F. Wrobel, "Detailed history of recoiling ions induced by nucleons," Computer Physics Communications, vol. 178, no. 2, pp. 88-104, Jan. 2008.

7. Available: http://www.srim.org/SRIM

8. B. D. Sierawski, K. M. Warren, R. A. Reed, R. A. Weller, M. M. Mendenhall, R. D. Schrimpf, R. C. Baumann, and V. Zhu, "Contri- bution of low-energy ( $\mathrm{MeV})$ neutrons to upset rate in a $65 \mathrm{~nm}$ SRAM," in Proc. IEEE Reliability Physics Symp., 2010, pp. 395-399.

9. G. M. Swift, G. R. Allen, C. W. Tseng, C. Carmichael, G. Miller, and J. S. George, "Static upset characteristics of the $90 \mathrm{~nm}$ virtex-4QV FPGAs," in Proc. IEEE Radiation Effects Data Workshop, 2008, pp. 98-105. 